\title{
IMPLICACIONES AMBIENTALES DE LAS TECNOLOGÍAS DE ENERGÍA RENOVABLE
}

\author{
Jan Mahirt-Smith \\ University of Toronto, Canada \\ jan.mahirt-s@rotman.utoronto.ca
}

(Tipo de artículo: INVESTIGACIÓN. Recibido el 01/07/2011. Aprobado el 20/09/2011)

\begin{abstract}
RESUMEN
Las tecnologías de energía renovable como la eólica, la solar y la biomasa, hacen un uso del suelo más intenso que las de combustibles fósiles tradicionales y, geográficamente, sus implicaciones ambientales son más heterogéneas, por lo que presentan un gran desafío para las técnicas de evaluación de su ciclo de vida. Este trabajo presenta los resultados de una investigación bibliográfica alrededor de los siguientes temas: 1) cambios en el uso del suelo debido a la mayor producción de energía renovable; 2) impactos del uso de suelo; 3) variabilidad geográfica en el inventario de datos; y 4) efectos de la distribución de energía. Además, se revisa el grado de investigación que actualmente se aplica acerca de las tecnologías de energía renovable en campos como el eólico, el solar y la bioenergía y en la evaluación del ciclo de vida en general.
\end{abstract}

\section{Palabras clave}

Biocombustibles, evaluación de ciclo de vida, energía solar, energía eólica, energía renovable, uso del suelo.

\section{ENVIRONMENTAL IMPLICATIONS OF RENEWABLE ENERGY TECHNOLOGIES}

\begin{abstract}
Renewable energy technologies like wind, solar and biomass use land in a more intense way than traditional fossil fuels and, geographically, its environmental implications are more heterogeneous, thus constituting a big challenge for the evaluation techniques of their life cycle. This work presents the results of a bibliographical research about these topics: 1) changes in land use because of increased production of renewable energy, 2) impacts of land use; 3) geographical variability in data inventory, and 4) effects of energy distribution. Besides, is reviewed the degree of research currently conducted on technologies of renewable energy in fields like wind, solar, and bioenergy and life cycle evaluation in general.
\end{abstract}

Keywords

Biofuels, life cycle assessment, solar power, wind power, renewable energy, land use.

\section{IMPLICATIONS ENVIRONNEMENTALES DES TECHNOLOGIES D'ÉNERGIE RENOUVELABLE}

\section{RÉSUMÉ}

Les technologies d'énergie renouvelable comme l'éolienne, la solaire et la biomasse, fait un usage du sol plus intense que ca des combustibles fossiles traditionnels et, géographiquement, ses implications environnementales sont plus hétérogènes, pour cette raison ils constituent un grand défi pour les techniques de son cycle de vie. Ce travail présent les résultats d'une recherche bibliographique sur les sujets suivants : 1) changements dans le usage du sol à cause de une production plus grande d'énergie renouvelable; 2) effets d'usage du sol; 3) variabilité géographique dans l'inventaire des donnés; et 4) effets de la distribution d'énergie. De manière supplémentaire, on vérifie le degré de recherche que dans l'actualité est appliqué au sujet des technologies d'énergie renouvelable dans domaines comme l'éolienne, le solaire et la bioénergie et dans l'évaluation du cycle de vie en général

\section{Mots-clés}

Biocombustibles, évaluation du cycle de vie, énergie solaire, énergie éolienne, énergie renouvelable, usage du sol. 


\section{INTRODUCCIÓN}

Debido a que se han incrementado las preocupaciones acerca de la sostenibilidad de una economía basada en combustibles fósiles, la investigación en sistemas de producción de energía renovable basados en biocombustibles también lo ha hecho, lo mismo que las políticas y el interés de la inversión. Sin embargo, las implicaciones ambientales y ecológicas del ciclo de vida de los sistemas renovables aún no se investigan satisfactoriamente. Las técnicas tradicionales de evaluación del ciclo de vida -LCA- como las codificadas por la ISO, todavía no se han desarrollado para sistemas con un fuerte componente geoespacial. Las primeras aplicaciones de LCA se realizaron en sistemas dependientes de combustibles fósiles, como automóviles y procesos químicos. El uso del suelo y las implicaciones geoespaciales de las tecnologías aplicadas en los sistemas de energía renovable representan un desafío para las bases de datos existentes, para los criterios de evaluación de impacto y para las representaciones matemáticas de variabilidad e incertidumbre.

Sin embargo, la creciente demanda por energía renovable es un factor particularmente importante del impacto de la modificación del uso del suelo, de las medioambientales o de las ecológicas. El rápido incremento de los precios del petróleo, la preocupación por el cambio climático global y la ampliación de las políticas en incentivos, han originado un nivel prácticamente sin precedentes en la investigación y en el interés comercial por las energías renovables. Entre las tecnologías de producción de este tipo de energía de más rápido crecimiento se encuentran la biomasa, la eólica y la solar. Mientras que los combustibles fósiles tradicionales generalmente están focalizados territorialmente, las tecnologías de energía renovable son más dispersas geográficamente; su rendimiento energético por unidad de superficie típicamente es más bajo y sus sistemas concomitantes de recolección, distribución y almacenamiento utilizan más intensivamente el suelo -por ejemplo, el transporte por carretera de camiones de etanol, comparado con los oleoductos y gasoductos. De forma general, las implicaciones del uso del suelo pueden ser mucho más profundas para las tecnología de biomasa, eólica y energía solar que para las basadas en combustibles fósiles.

Las implicaciones ambientales del uso del suelo han sido el foco de una intensa, aunque dispar, atención de la comunidad investigativa en todo el mundo y han originado una serie de nuevos estudios relacionados con las implicaciones del ciclo de vida del uso del suelo por parte de las tecnologías de energía alternativa. Las contribuciones provienen desde un conjunto diverso de disciplinas científicas, incluyendo la ciencia atmosférica, la economía y la ecología industrial. Una cosa que tienen en común estas contribuciones es la comprensión de que el ciclo de vida es la perspectiva adecuada para el análisis técnico y político, sin embargo, las tecnologías de la biomasa, el viento y la energía solar tienen importantes implicaciones técnicas, ambientales, de uso de suelo y geoespaciales que rara vez se abordan directamente en los proceso de LCA. Por ejemplo, la agricultura de la materia prima para el etanol, las grandes plantas de energía solar y las "granjas" de viento, tienen un mayor grado de variabilidad y heterogeneidad geográfica del que pueden suministrar los actuales métodos de LCA. En consecuencia, existe una serie de nuevas temáticas relacionadas con el uso del suelo que cada vez son más urgentes desde la perspectiva de las políticas de la información y en el diseño y la toma de decisiones ambientales, pero que no se abordan adecuadamente en las técnicas actuales de evaluación del ciclo de vida. Con el fin de evaluar apropiadamente los impactos de la energía renovable, los métodos deben desarrollarse para:

1. Cambios en el modelo de utilización de los recursos del suelo; por ejemplo, en el cultivo de tierras forestales para combustibles.

2. Caracterizar los impactos del uso del suelo utilizando métricas adecuadas; por ejemplo, en la pérdida de la biodiversidad.

3. Comprender la variabilidad geográfica del inventario de datos del ciclo de vida; por ejemplo, las emisiones de nitrógeno desde los diferentes suelos y los factores de caracterización.

4. Efectos de los modelos de transporte y de distribución de energía; por ejemplo, la construcción de vías de acceso y de la red eléctrica para los sitios remotos a las fuentes solares 0 eólicas y/o efectos del transporte de la biomasa y los biocombustibles.

Este trabajo identifica esas cuatro áreas como lagunas de investigación críticas e importantes relacionadas con los aspectos geoespaciales y de uso del suelo en la evaluación del ciclo de vida de las tecnologías de energía renovable.

\section{LAGUNAS DE INVESTIGACIÓN}

\subsection{Cambios en la utilización del suelo}

El enfoque para LCA basado en procesos, generalmente no tiene en cuenta la utilización de recursos, salvo en el caso especial del agotamiento de los combustibles fósiles o, más raramente, del área total del suelo dedicado a una función en particular [1]; sin embargo, el significado de "consumo" en términos de uso del suelo no es claro. A diferencia de los combustibles fósiles, la calidad del suelo puede ser degradada o restaurada dependiendo de la forma en que se utilice. Predecir cambios en el uso del suelo como una función de la demanda final requiere una extensión de las técnicas tradicionales de LCA para incluir el conocimiento externo a las mismas, como el modelado basado en agentes, los Sistemas de Información Geográfica, la economía y la dinámica de sistemas. La necesidad de aplicar estos métodos para evaluar las energías renovables es particularmente grave. La migración desde la producción basada en combustibles fósiles, a la basada en recursos renovables, requerirá cambios masivos en los patrones 
de uso del suelo, por ejemplo, es probable que se incremente la superficie total de suelos cultivados cuando las tierras, previamente forestadas 0 marginales, se incorporen a la producción de cultivos energéticos. El suelo del desierto probablemente se utilice para la producción de energía solar térmica o fotovoltaica, mientras que las turbinas eólicas se ubicarán en una variedad de lugares -algunos de ellos sensitivos ecológica, social, ambiental e incluso políticamente.

\subsection{Caracterización del impacto del uso del suelo}

Las potenciales implicaciones de los cambios de uso del suelo son mucho más complejas para caracterizar cuáles se pueden capturar en las métricas de uso común, como el calentamiento global o la potencial eutrofización [2-4]. Los efectos del cambio de uso del suelo puede influir en el cambio climático desde la liberación de carbono en el suelo y/o cambios en los patrones de evapotranspiración, la destrucción del hábitat ecológico, la apertura de áreas remotas para la construcción y el mantenimiento de infraestructuras, los cambios en la migración del polvo, la erosión del suelo y los patrones de viento locales o regionales. Por lo tanto, se deben caracterizar las implicaciones del ciclo de vida del uso del suelo para energías renovables mediante un conjunto de datos más diverso del que se expresa en LCA, incluyendo el incremento del carbono, las emisiones de nitrógeno o fósforo, la pérdida de la biodiversidad y otros servicios del ecosistema, la degradación del suelo o la calidad del agua y los efectos en el cambio climático de los gases de no-efecto invernadero a escala local, regional o, incluso, mundial. Ya que el uso del suelo para la producción de energía implica más que simplemente liberar contaminantes químicos en el medio ambiente, se requiere identificar y definir un conjunto de métricas apropiadas para los servicios perdidos de los ecosistemas, que la tierra podría proporcionar de otra forma. Es poco probable que alguna métrica individual pueda ser satisfactoria.

\subsection{La variabilidad geográfica}

Los enfoques tradicionales de LCA suponen grandes y centralizadas instalaciones de fabricación 0 de producción. En estos casos, las estimaciones puntuales con límites de incertidumbre, como los que reporta el Monte Carlo Analysis, son apropiados para la caracterización del inventario de datos. Sin embargo, en comparación con los sistemas de producción basados en combustibles fósiles, la producción de energía renovable es muy dependiente del lugar. Es decir, es probable que los inventarios de emisiones tengan una amplia distribución con base en factores como la calidad del suelo local, la precipitación, el viento u otros problemas climáticos, o incluso a factores socio-políticos como las prácticas de gestión. La incertidumbre puede ser tan alta que, en algunos casos, incluso el signo -positivo o negativode la propia representación del inventario de datos pueda ser cuestionado. La desagregación de variabilidad y de incertidumbre para los recursos renovables resultará en una representación más exacta. Sin embargo, esto requiere un conocimiento especializado de los parámetros y las relaciones funcionales que, típicamente, están por fuera del ámbito tradicional de LCA. Por ejemplo, la deforestación para producir cultivos energéticos en las regiones tropicales, tendrá un impacto significativamente diferente en el uso del suelo que el cultivo perenne de céspedes de verano en las zonas templadas. Los LCA necesitan capturar esta variabilidad, debido a que una simple cuantificación de la superficie ocupada por la biomasa no puede relacionar la degradación de los recursos del suelo. Del mismo modo, el hecho de agregar inventario de datos a los impactos del punto medio, como la formación de smog, la eutrofización, o la salud humana, requieren el conocimiento de los factores de caracterización que representan equivalencias de punto medio. En el caso de las cuestiones ambientales globales -como el potencial calentamiento global por gases de efecto invernadero 0 el potencial agotamiento de la capa de ozono por CFCs y sus sustitutos- los factores de caracterización estandarizada, generalmente, son bien estimados. Sin embargo, los puntos medios con una ubicación local o regional tienen un mayor grado de variabilidad. Por ejemplo, los puntos medios en la salud humana dependen altamente de la proximidad geográfica de las emisiones a los receptores humanos, así como las condiciones locales de destino y de transporte. Del mismo modo, la química medioambiental local puede jugar un papel críticamente importante, en tanto que el smog -es decir, el ozono troposférico-, la formación y la eutrofización, dependen de que las especies químicas en un área local sean el precursor o los nutrientes limitantes. Actualmente, los datos de variabilidad geográfica, como el inventario de datos y los factores de caracterización, están representados pobremente en el LCA.

\subsection{El transporte de la energía y los efectos de su distribución}

Los LCA tradicionales pueden incluir en su marco a las distancias para el transporte, pero la energía renovable presenta problemas logísticos y de infraestructura que no abordan. La naturaleza geográficamente dispersa de los recursos de energías renovables plantea nuevos problemas para la recolección y distribución de energía [5]. Por ejemplo, típicamente las materias primas de biomasa son voluminosas y húmedas comparadas con los recursos basados en combustibles fósiles-y poco rentables para transportar a largas distancias [6]. El etanol no puede viajar en los gasoductos existentes y los recursos de energía eólica y solar pueden ser más abundantes en zonas donde no existe alta capacidad en la infraestructura para distribución eléctrica. En consecuencia, los sistemas de energía renovable probablemente requieran una redistribución de la infraestructura, incluyendo caminos nuevos, ferrocarriles, puertos y/o líneas de transmisión eléctrica. Por otra parte, los actuales aspectos geoespaciales de los flujos de energía pueden evolucionar hasta involucrar algunas áreas de intercambio para importadores y exportadores de 
energía y viceversa. Es necesario comprender los aspectos geoespaciales sistémicos de la energía renovable, en lo que tiene que ver con el transporte y los sistemas de colección/distribución de energía, para crear una imagen completa de los aspectos ambientales del ciclo de vida de la energía renovable. Sin embargo, los enfoques actuales de LCA rara vez intentan comprender los efectos explícitos del transporte geográficamente, aunque sí incluyen estimaciones muy globales del incremento de la actividad de transporte.

\section{METODOLOGÍA DE LA INVESTIGACIÓN}

Para comprender el grado en que se estudian los problemas de LCA de las energías renovables, se llevó a cabo una revisión de la literatura científica. Con base en el contenido de los resúmenes de cada artículo relevante, publicado en el índice electrónico scholar.google.com y en la Web of Science, se clasificaron como pertenecientes a una de las cuatro categorías descritas anteriormente: cambios en el uso del suelo, calidad del inventario de datos, variabilidad del factor de caracterización, o aspectos de transporte/distribución. En cada categoría también se tuvo en cuenta a los artículos de revistas que abordan temas múltiples. Además, se codificaron con respecto a sí trataban la tecnología de biomasa, la solar, la eólica o los LAC en general. Debido a que los índices utilizados no son exhaustivos, es posible que algunas publicaciones no se hayan incluido, por lo tanto, los resultados se deben interpretar como una base comparativa, es decir, como una medida relativa de interés para la investigación.

\section{RESULTADOS OBTENIDOS Y DISCUSIÓN}

La Tabla 1 muestra los resultados de la revisión de la literatura en la que se investiga la intersección de las tecnologías y los métodos LCA. En cada celda de la tabla se detalla el número de publicaciones en revistas arbitradas en la literatura reciente y que abordan una cuestión metodológica específica con respecto a una tecnología específica. En total, se revisaron 71 trabajos arbitrados en las cuatro categorías definidas y en cada una de las tres tecnologías de energía renovable, o LCA en general -es decir, métodos de análisis geoespacial desarrollados en artículos que no especifican la tecnología-energía.

Tabla 1

Número de publicaciones recientes por categoría y tecnología de LCA

\begin{tabular}{|c|c|c|c|c|}
\hline & Biomasa & Eólica & Solar & LCA General \\
\hline Modelado del cambio de uso de suelo [7-41] & 9 & 2 & 1 & 14 \\
\hline Calidad del inventario de datos: variabilidad e incertidumbre [1, 2, 42-59] & 11 & 2 & 2 & 7 \\
\hline Caracterización geográfica del factor de variabilidad [60-63] & 13 & 0 & 2 & 3 \\
\hline Aspectos de transportación: demandas y emisiones [64-68] & 3 & 0 & 0 & 2 \\
\hline
\end{tabular}

Como era de esperar, la mayoría de los estudios revisados, 37, se publicaron en revistas enfocadas en la evaluación del ciclo de vida, en la ecología industrial, o en revistas que tienen una amplia agenda ambiental; 22 de los artículos se publicaron en revistas relacionadas con la energía. El resto de estudios proviene de una amplia variedad de revistas disciplinarias, con tópicos que van desde la agronomía, la hidrología, la geografía, el paisaje y la planificación urbana, hasta la logística. Esta revisión destaca el carácter multidisciplinar de la investigación en el cambio de uso del suelo y la diversidad y complejidad de la investigación en esta área.

La Tabla 1 muestra una gran cantidad de actividades de investigación en aspectos de uso del suelo de LCA para aplicaciones de biomasa, que era de esperar, dada la reciente expansión de la industria de los biocombustibles en respuesta a las nuevas iniciativas de política gubernamental. Los datos acerca de LCA para las tecnologías eólica y solar son escasos, particularmente con respecto a las implicaciones del uso del suelo; sin embargo, estas tecnologías pueden tener importantes repercusiones a este respecto, como cambios en los patrones de la evapotranspiración, la ocupación de tierras por las turbinas, las vías de acceso y el equipo de transmisión, o cambios en la idoneidad del hábitat en o cerca a los sitios de la turbina [69]. Esta es un área potencial de investigación que se debe abordar en el futuro Además, en la literatura, se discuten los métodos LCA generales con énfasis específico en cómo modelar el cambio de uso del suelo y las adecuadas métricas de impacto a utilizar; sin embargo, estos métodos no se han aplicado ampliamente a las tecnologías de energía renovable que no sean de origen biológico.

La mayoría de las investigaciones se centran en determinar métricas adecuadas para el cambio de uso del suelo y la calidad del inventario de datos. Se encontró una diversidad de opiniones en relación a cómo evaluar adecuadamente los impactos del cambio de uso del suelo. Los métodos propuestos varían enormemente: van desde la estimación de la biodiversidad hasta el análisis exergético para estimar la productividad del suelo para calcular la huella ecológica. Aunque ésta es el área del cambio de uso del suelo en LCA que ha recibido la mayor atención en las investigaciones, no se ha llegado a un consenso para determinar el método más adecuado para medir sus impactos.

Las dos últimas categorías que se trataron en este estudio, la caracterización geográfica y los aspectos de transporte, no se abordan de manera integral en la mayoría de los estudios centrados en la bioenergía. La ubicación de las tecnologías de energía eólica y solar en zonas ecológicamente sensibles merece atención, sobre todo teniendo en cuenta que la disponibilidad de los recursos eólicos y solares, a menudo, está 
desconectada de la red de la infraestructura eléctrica, donde se necesita esa energía. Los problemas de distribución y la construcción de infraestructura adicional son dos aspectos de las tecnologías de energía renovable que se deben abordar más ampliamente.

Existe relativamente pocos estudios de las implicaciones del uso del suelo de las tecnologías eólica y solar, a pesar de que el uso del suelo puede ser un aspecto importante de su implementación a gran escala. En los últimos años, el Bureau of Land Management de los EEUU ha recibido un aluvión de solicitudes de permisos para nuevas instalaciones en ambas tecnologías y, recientemente, tuvo que crear el Departmental Task Force on Energy and Climate Change para desarrollar una estrategia para su revisión [70]. Varios de estos proyectos proponen una escala sin precedentes de desarrollo. Un senador americano anunció planes para una legislación que limite la recolección y la distribución de recursos energéticos renovables en tierras federales [71]. Sin embargo, en el caso de la energía geotermal, recientemente se designaron más de 190 millones de acres de tierras federales para el desarrollo o el transporte de nuevos proyectos de ese tipo de energía [72].

\section{CONCLUSIONES}

Las preocupaciones por los impactos del uso del suelo de las energías renovables, en el ámbito de la política, contrastan con la escasa actividad de investigación científica en este campo -especialmente en el transporte y la distribución de la energía eólica y solar, en comparación con la bioenergía. Esta diferencia sugiere que se debe promover una mayor actividad de investigación; pero, debido a la diversidad de perspectivas disciplinares, los métodos de investigación y los límites en relación con este complejo asunto, se requiere una mayor discusión para ayudar a determinar las direcciones apropiadas de la investigación y para crear consenso.

Para tal fin, la National Science Foundation patrocinó, en 2009, el Workshop on Land Use and Geospatial Aspects of Renewable Energy. Su objetivo fue promover el intercambio entre expertos en LCA y expertos en tecnología específica que están trabajando en el campo de las energías renovables. Los grupos de trabajo conformados trataron cuestiones metodológicas y tecnológicas. Este evento se llevó a cabo en conjunción con la conferencia Internacional Life Cycle Assessment IX, para garantizar a los profesionales e investigadores en LCA una mayor visibilidad y accesibilidad a las cuestiones de uso del suelo.

Se espera que de estos encuentros surjan respuestas a las necesidades identificadas en las lagunas de la investigación que merecen mayor atención, lo mismo que para la creación de una comunidad diversa de investigadores preparados para responder acerca del uso del suelo en los LCA de las energías renovables.

\section{REFERENCIAS}

[1] M. Stewart \& B. Weidema. "A consistent framework for assessing the impacts from resource use - A focus on resource functionality". International Journal of Life Cycle Assessment, Vol. 10, No. 4, pp. 240-247. 2005.

[2] F. Brentrup et al. "Life cycle impact assessment of land use based on the hemeroby concept". International Journal of Life Cycle Assessment, Vol. 7, no. 6, pp. 339348. 2002.

[3] S. J. Cowell \& R. Clift. "A methodology for assessing soil quantity and quality in life cycle assessment". Journal of Cleaner Production, Vol. 8, No. 4, pp. 321-331. 2000.

[4] M. Lenzen \& S. A. Murray. "A modified ecological footprint method and its application to Australia". Ecological Economics, Vol. 37, No. 2, pp. 229-255. 2001.

[5] G. Forsberg. "Biomass energy transport Analysis of bioenergy transport chains using life cycle inventory method". Biomass and Bioenergy, Vol. 19, No. 1, pp. 1730. 2000.

[6] C. Hamelinck; R. A. A. Suurs \& A. P. C. Faaij. "International bioenergy transport costs and energy balance". Biomass and Bioenergy, Vol. 29, No. 2, pp. 114-134. 2005.

[7] T. Wagendorp et al. "Land use impact evaluation in life cycle assessment based on ecosystem thermodynamics". Energy, Vol. 31, No. 1, pp. 112-125. 2006.

[8] J. Fargione et al. "Land clearing and biofuel carbon debt". Science, Vol. 319, No. 5867, pp. 1235-1238. 2008.

[9] T. Searchinger et al. "Use of U.S. croplands for biofuels increases greenhouse gases through emissions from land-use change". Science, Vol. 319, No. 5867, pp. 1238-1240. 2008.

[10] R. Lal \& J. P. Bruce. "The potential of world cropland soils to sequester $C$ and mitigate the greenhouse effect". Environmental Science \& Policy, Vol. 2, No. 2, pp. 177185. 1999.

[11] R. D. Perlack et al. "Biomass as Feedstock for a Bioenergy and Bioproducts Industry: The Technical Feasibility of a Billion-Ton Annual Supply". Technical reptort number A357634, for USDA and US DOE, Washington. 2005.

[12] B. F. Zhan et al. "A GIS-enabled comparison of fixed and discriminatory pricing strategies for potential switchgrass-to-ethanol conversion facilities in Alabama". Biomass and Bioenergy, Vol. 28, No. 3, pp. 295-306. 2005.

[13] G. Stoglehner. "Ecological footprint: A tool for assessing sustainable energy supplies". Journal of Cleaner Production, Vol. 11, No. 3, pp. 267-277. 2003.

[14] W. Krewitt \& J. Nitsch. "The potential for electricity generation from on-shore wind energy under the constraints of nature conservation: a case study for two regions in Germany". Renewable Energy, Vol. 28, No. 10, pp. 1645-1655. 2003.

[15] A. Antón; F. Castells \& J. I. Montero. "Land use indicators in life cycle assessment. Case study: The environmental impact of mediterranean greenhouses". Journal of Cleaner Production, Vol. 15, No. 5, pp. 432438. 2007.

[16] H. Blonk; E. Lindeijer \& J. Broers. "Towards a methodology for taking physical degradation of ecosystems into account in LCA". The International Journal of Life Cycle Assessment, Vol. 2, No. 2, pp. 9198. 1997.

[17] F. Brentrup et al. "Environmental impact assessment of agricultural production systems using the life cycle 
assessment methodology I. Theoretical concept of a LCA method tailored to crop production". European Journal of Agronomy, Vol. 20, No. 3, pp. 247-264. 2004.

[18] L. M. I. Canals et al. "Key elements in a framework for land use impact assessment within LCA". International Journal of Life Cycle Assessment, Vol. 12, No. 1, pp. 515. 2007.

[19] L. M. I. Canals; J. Romanya \& S. J. Cowell. "Method for assessing impacts on life support functions (LSF) related to the use of 'fertile land' in life cycle assessment (LCA)". Journal of Cleaner Production, Vol. 15, No. 15, pp. 14261440. 2007

[20] J. Dewulf et al. "Cumulative exergy extraction from the natural environment (CEENE): A comprehensive life cycle impact assessment method for resource accounting". Environmental Science and Technology, Vol. 41, No. 24, pp. 8477-8483. 2007.

[21] J. Dewulf et al. "Exergy: Its potential and limitations in environmental science and technology". Environmental Science and Technology, Vol. 42, No. 7, pp. 2221-2232. 2008.

[22] V. Fthenakis \& H. C. Kim. "Land use and electricity generation: A life-cycle analysis". Renewable and Sustainable Energy Reviews", Vol. 13, No. 6-7, pp. 1465-1474. 2009.

[23] J. L. Hau \& B. R. Bakshi. "Expanding exergy analysis to account for ecosystem products and services". Environ Sci Technol, Vol. 38, No. 13, pp. 3768-3777. 2004.

[24] K. Hedegaard; K. A. Thyø \& H. Wenzel. "Life cycle assessment of an advanced bioethanol technology in the perspective of constrained biomass availability". Environmental Science and Technology, Vol. 42, No. 21, pp. 7992-7999. 2008.

[25] M. A. J. Huijbregts. "Part II: Dealing with parameter uncertainty and uncertainty due to choices in life cycle assessment". The International Journal of Life Cycle Assessment, Vol. 3, No. 6, pp. 343-351. 1998.

[26] D. Styles \& M. B. Jones. "Energy crops in Ireland: Quantifying the potential life-cycle greenhouse gas reductions of energy-crop electricity". Biomass \& Bioenergy, Vol. 31, No. 11, pp. 759-772. 2007.

[27] J. Barrett \& A. Scott. "The Ecological Footprint: A Metric for Corporate Sustainability". Corporate Environmental Strategy, Vol. 8, No. 4, pp. 316-325. 2001.

[28] N. T. Hoagland. "Non-traditional tools for LCA and sustainability". International Journal of Life Cycle Assessment, Vol. 6, No. 2, pp. 110-117. 2001.

[29] J. G. Vogtlander et al. "Characterizing the change of land-use based on flora: application for EIA and LCA". Journal of Cleaner Production, Vol. 12, No. 1, pp. 47-57. 2004.

[30] R. Muller-Wenk. "Land Use - The Main Threat to Species: How to Include Land Use in LCA". IWÖDiskussionbeitrag, No. 64, pp. 1-46. 1998.

[31] F. Brentrup et al. "Life cycle impact assessment of land use based on the hemeroby concept". International Journal of Life Cycle Assessment, Vol. 7, No. 6, pp. 339348. 2002.

[32] E. Lindeijer. "Biodiversity and life support impacts of land use in LCA". Journal of Cleaner Production, Vol. 8, No. 4, pp. 313-319. 2000.

[33] J. W. Owens. "Water resources in Life-Cycle Impact Assessment: Considerations in choosing category indicators". Journal of Industrial Ecology, Vol. 5, No. 2, pp. 37-54. 2001.

[34] R. van den Broek et al. "Green energy or organic food? A life-cycle assessment comparing two uses of set-aside land". Journal of Industrial Ecology, Vol. 5, No. 3, pp. 6587. 2001.
[35] T. Köllner. "Species-pool effect potentials (SPEP) as a yardstick to evaluate land-use impacts on biodiversity". Journal of Cleaner Production, Vol. 8, No. 4, pp. 293311. 2000.

[36] G. F. Haas; F. Wettterich \& U. Geier. "Life Cycle Assessment Framework in agriculture on the farm level". International Journal of Life Cycle Assessment, Vol. 5, No. 6, pp. 345-348. 2000.

[37] K. Andersson et al. "The feasibility of including sustainability in LCA for product development". Journal of Cleaner Production, Vol. 6, No. 3-4, pp. 289-298. 1998.

[38] C. Simmons; K. Lewis \& J. Barrett. "Two feet - two approaches: a component-based model of ecological footprinting". Ecological Economics, Vol. 32, pp. 375380. 2000.

[39] B. Moller. "Changing wind-power landscapes: regional assessment of visual impact on land use and population in Northern Jutland, Denmark". Applied Energy. Vol. 83, No. 5, pp. 477-494. 2006.

[40] T. Tsoutsos; N. Frantzeskaki \& V. Gekas. "Environmental impacts from the solar energy technologies". Energy Policy, Vol. 33, No. 3, pp. 289296. 2005.

[41] L. Gagnon; C. Bélanger \& Y. Uchiyama. "Life-cycle assessment of electricity generation options: The status of research in year 2001". Energy Policy, Vol. 30, No. 14, pp. 1267-1278. 2002.

[42] J. H. Schmidt. "Development of LCIA characterisation factors for land use impacts on biodiversity". Journal of Cleaner Production, Vol. 16, No. 18, pp. 1929-1942. 2008.

[43] M. A. J. Huijbregts et al. "Framework for Modeling Data Uncertainty in Life Cycle Inventories". The International Journal of Life Cycle Assessment, Vol. 6, No. 3, pp. 127132. 2001.

[44] G. Berndes; M. Hoogwijk \& R. van den Broek. "The contribution of biomass in the future global energy supply: a review of 17 studies". Biomass and Bioenergy, Vol. 25, No. 1, pp. 1-28. 2003.

[45] A. E. Landis; S. A. Miller \& T. L. Theis. "Life cycle of the corn-soybean agroecosystem for biobased production". Environmental Science and Technology, Vol. 41, No. 4, pp. 1457-1464. 2007.

[46] B. Arheimer \& R. Liden. "Nitrogen and phosphorus concentrations from agricultural catchments-influence of spatial and temporal variables". Journal of Hydrology, Vol. 227, No. 1-4, pp. 140-159. 2000.

[47] V. Dornburg; I. Lewandowski \& M. Patel. "Comparing the land requirements, energy savings, and greenhouse gas emissions reduction of biobased polymers and bioenergy: An analysis and system extension of lifecycle assessment studies". Journal of Industrial Ecology, Vol. 7, No. 3-4, pp. 93-116. 2003.

[48] F. Field; R. Kirchain \& J. Clark. "Life-cycle assessment and temporal distributions of emissions". Journal of Industrial Ecology, Vol. 4, No. 2, pp. 71-91. 2000.

[49] P-A. Jacinth; R. L. Owens \& D. L. Hothem. "Transport of labile carbon in runoff as affected by land use and rainfall characteristics". Soil \& Tillage Research, Vol. 77, No. 2, pp. 111-123. 2004.

[50] B. Mattsson; C. Cederberg \& L. Blix. "Agricultural land use in life cycle assessment (LCA): case studies of three vegetable oil crops". Journal of Cleaner Production, Vol. 8, No. 4, pp. 283-292. 2000.

[51] T. O. West \& G. Marland. "A synthesis of carbon sequestration, carbon emissions, and net carbon flux in agriculture: comparing tillage practices in the United States". Agriculture, Ecosystems \& Environment, Vol. 91, No. 1-3, pp. 217-232. 2002. 
[52] T. O West \& G. Marland. "Net carbon flux from agriculture: Carbon emissions, carbon sequestration, crop yield, and land-use change". Biogeochemistry, Vol. 63, No.1, pp. 73-83. 2003.

[53] T. O. West \& N. Peña. "Determining thresholds for mandatory reporting of greenhouse gas emissions". Environmental Science and Technology, Vol. 37, No. 6, pp. 1057-1060. 2003.

[54] W. W. Wilhelm et al. "Crop and soil productivity response to corn residue removal: A literature review". Agronomy Journal, Vol. 96, No. 1, pp. 1-17. 2004.

[55] C. Wagner-Riddle \& G. W. Thurtell. "Nitrous oxide emissions from agricultural fields during winter and spring thaw as affected by management practices". Nutrient Cycling in Agroecosystems, Vol. 52, No. 2-3, pp. 151-163. 1998.

[56] R. P. Udawatta; P. P. Motavalli \& H. E. Garrett. "Phosphorus loss and runoff characteristics in three adjacent agricultural watersheds with claypan soils". Journal of Environmental Quality, Vol. 33, No. 5, pp. 1709-1719. 2004.

[57] S. A. Miller; A. E. Landis \& T. L. Theis. "Use of Monte Carlo Analysis to Characterize Nitrogen Fluxes in Agroecosystems". Environ. Sci. Technol. Vol. 40, No. 7, pp. 2324-2332. 2006.

[58] R. F. Dones \& R. Frischknech. "Life-cycle assessment of photovoltaic systems: results of Swiss studies on energy chains". Progress in Photovoltaics: Research and Applications, Vol. 6, No. 2, pp. 117-125. 1998.

[59] M. Lenzen. "Energy and CO2 life-cycle analyses of wind turbines-review and applications". Renewable Energy, Vol. 26, No. 3, pp. 339-362. 2002.

[60] A. D. Hartkamp; J. W. White \& G. Hoogenboom. "Interfacing geographic information systems with agronomic modeling: A review". Agronomy Journal, Vol. 91, No. 5, pp. 761-772. 1999.

[61] R. L. Graham; B. C. English \& C. E. Noon. "A Geographic Information System-based modeling system for evaluating the cost of delivered energy crop feedstock". Biomass and Bioenergy, Vol. 18, No. 4, pp. 309-329. 2000.
[62] S. M. J. Baban \& T. Parry. "Developing and applying a GIS-assisted approach to locating wind farms in the UK". Renewable Energy, Vol. 24, No. 1, pp. 59-71. 2001.

[63] I. H. Knoepfel. "A framework for environmental impact assessment of long-distance energy transport systems". Energy, Vol. 21, No. 7-8, pp. 693-702. 1996.

[64] S. Abukhader \& G. Jönson. "Logistics and the environment: Is it an established subject?" International Journal of Logistics, Vol. 7, No. 2, pp. 137-149. 2004.

[65] A. Caputo et al. "Economics of biomass energy utilization in combustion and gasification plants: effects of logistic variables". Biomass and Bioenergy, Vol. 28, No. 1, pp. 35-51. 2005.

[66] J. G. Vogtländer et al. "Characterizing the change of land-use based on flora: Application for EIA and LCA". Journal of Cleaner Production, Vol. 12, No. 1, pp. 47-57. 2004.

[67] T. Wagendorp et al. "Land use impact evaluation in life cycle assessment based on ecosystem thermodynamics". Energy, Vol. 31, No. 1, pp. 112-25. 2006.

[68] C. Wagner-Riddle \& G. W. Thurtell. "Nitrous oxide emissions from agricultural fields during winter and spring thaw as affected by management practices". Nutrient Cycling in Agroecosystems, Vol. 52, No. 2-3, pp. 151-63. 1998.

[69] National Research Council. "Environmental Impacts of Wind-Energy Projects". The National Academy Press. 2008.

[70] The Secretary of the Interior. "Renewable Energy Development by the Department of the Interior". Secretarial Order \#3285. 2009.

[71] L. Greenemeier. "Cruel irony: Do renewable power plants threaten their surrounding environment?" 2009.

[72] Bureau of Land Management. "Record of Decision and Resource Management Plan Amendments for geothermal leasing in the Western United States". BLMWO-GI-09-003-1800. 2008. 